\title{
Fibroadenoma in the axillary accessory breast
}

\author{
M Motsumi ${ }^{1}$, M Narasimhamurthy², M Gabolwelwe ${ }^{2}$ \\ ${ }^{1}$ Department of Surgery, University of Botswana, Sir Ketumile Masire Hospital, Gaborone, Botswana \\ ${ }^{2}$ Department of Pathology, University of Botswana, Sir Ketumile Masire Hospital, Gaborone, Botswana
}

Corresponding author: Mpapho Joseph Motsumi (josephmotsumi@yahoo.com)

\begin{abstract}
Summary: A 30-year-old female noted a mass in her right axilla for 6 years. The mass was painless, slowly growing, causing discomfort, had cyclical changes in consistency and was of cosmetic concern to the patient. Examination revealed normal breasts and a soft, non-tender, broad based mass in the right axilla. Sonography was non-diagnostic. Diagnostic doubt, cosmetic concern and discomfort to the patient prompted excisional biopsy which revealed a $1 \mathrm{~cm}$ fibroadenoma with surrounding breast tissue and a diagnosis of fibroadenoma arising in accessory breast tissue was made. Surgical excision is recommended for symptomatic accessory breast tissue where diagnostic doubt exists and for cosmetic reasons.
\end{abstract}

Keywords: Fibroadenoma in Supernumerary breast, Fibroadenoma in Ectopic breast, Fibroadenoma in Accessory breast, Fibroadenoma in polymastia.

S Afr J Surg 2018;56(3) http://dx.doi.org/10.17159/2078-5151/2018/v56n3a2444

\section{Case Report}

A 30-year-old female presented at our outpatient surgical clinic at Princess Marina Hospital, Gaborone, Botswana. She reported noting a mass in her right axilla for 6 years. The mass was painless, slowly growing and had cyclical monthly changes in consistency. She reported some discomfort and cosmetic concerns. The contralateral axilla was unaffected. There was no family history of breast cancer. Breast examination was normal. Under the right axilla a soft, non-tender, mobile broad based $4 \times 3 \mathrm{~cm}$ mass of uniform consistency was palpable. There was no nipple or areola on the skin overlying the mass. An ultrasound reported "A masslike lesion with mixed echogenicity", findings compatible with either a lipoma or an accessory breast. In view of the diagnostic uncertainty, discomfort and cosmetic concerns from the patient, an excisional biopsy was performed. Intraoperative findings were that of a fibro-fatty tissue with a small $1 \mathrm{~cm}$ nodule which was thought to be a lymph node. Histopathological examination of the nodule showed a well-circumscribed lesion consisting of tubular and slit-like spaces lined by epithelial and myoepithelial cells. Stroma showed myxomatous changes. These histological features were consistent with a fibroadenoma. Adjacent tissue showed mainly mature adipocytes admixed with few terminal duct lobular units. These histological features are consistent with a fibroadenoma arising from accessory breast tissue. The patient's recovery was uneventful and she was informed about the results and reassured.

\section{Discussion}

Accessory breast(s) is a term used to describe the presence of more than two breasts in human beings. It is synonymous to supernumerary breast, ectopic breast and polymastia. Accessory breasts have the potential to undergo both benign and non-benign transformations as normal breast tissue. ${ }^{1-6}$ Accessory breasts occur along the milk line extending from the axilla to the pubic region. ${ }^{1-6}$ This line represents the location of the embryonic mammary ridges which involutes except for the two segments located in the pectoral regions which develop into breasts. Accessory breasts develop when there is failure of the ridges to involute. Accessory breasts may also develop outside the milk line. A very rare case was recently reported in a 15-year-old Indian female who had two inter-scapular accessory breasts with two nipples and seven areolae. ${ }^{1}$

The incidence of accessory breast is $1-6 \%$ of the normal population. ${ }^{1,3,4}$

Cases of accessory breasts are usually sporadic but familial presentations associated with urogenital and congenital cardiac abnormalities have been described although the association has been challenged. ${ }^{4}$

Kajava developed the accessory breast classification system in 1915 which is still being used today ${ }^{3}$ (Table 1).

Surgical excision is recommended if they are symptomatic, of cosmetic concern or diagnostic doubt exists.

Fewer than forty cases of fibroadenoma in accessory breasts have been reported in the literature. ${ }^{2}$ We searched and reviewed 29 reported cases of fibroadenoma in accessory breasts. The age of reported cases ranged from 14-42 years. The average 


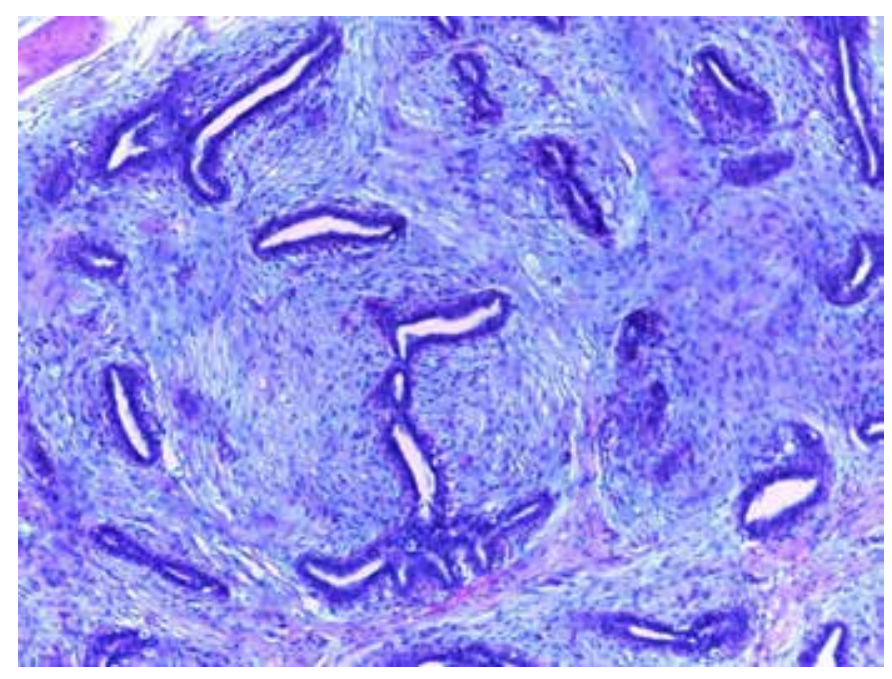

Figure 1

\section{Table 1: Kajava Classification}

Class I consists of a complete breast including glandular tissue, nipple, and areola.

Class II consists of only glandular tissue and nipple, without areola.

Class III consists of only glandular tissue and areola, without nipple.

Class IV consists of only glandular tissue.

Class V (pseudomamma) consists of only nipple and areola, without glandular tissue.

Class VI (polythelia) consists of only the nipple.

Class VII (polythelia areolaris) consists of only the areola.

Class VIII (polythelia pilosa) consists of only hair.

diameter of these fibroadenomas is $1.4 \mathrm{~cm}$. The anatomical locations of the accessory breasts were: 21 in the axilla, 6 in the vulva, and 1 at the back. Not all fibroadenomas in the accessory breast are palpable and hence a careful examination and sonographic assessment is essential. In our case, the fibroadenoma was not palpable and ultrasound assessment was inconclusive. A lump was identified intraoperatively but was confused for a lymph node. Hence, it is important to send the specimen for histopathological examination to exclude non-benign pathologies.

Accessory breast(s) are a rare occurrence which should be considered as a differential diagnosis of masses occurring along the milk line, although they can occur at other sites. Benign and non-benign breast pathologies can occur in ectopic breast tissue. The treatment of choice when the lump is symptomatic, unsightly or there is diagnostic doubt is excision and histological examination to exclude other pathologies.

\section{REFERENCES}

1. Shreshtha S. Supernumerary Breast on the Back: A Case Report. Indian J Surg. 2016;78(2):155-7. doi:10.1007/s12262016-1443-8

2. Surd A, Mironescu A, Gocan H. Fibroadenoma in Axillary Supernumerary Breast in a 17-Year-Old Girl: Case Report. J Pediatr Adolesc Gynecol. 2016;29(5):e79-e81. doi:10.1016/j. jpag.2016.04.008

3. Vidyasagar R, Singh RN. A Rare Presentation of An Ectopic Breast Tissue In Axilla. Pol Przegl Chir. 2015;87(10):528-30. doi:10.1515/pjs-2015-0099

4. Tiwary SK, Kumar P, Khanna AK. Fibroadenoma in axilla: another manifestation of ectopic breast. BMJ Case Rep. 2015;2015. doi:10.1136/bcr-2015-209535

5. Ayadi-Kaddour A, Khadhar A, Mlika M, et al. Fibroadenoma in an ectopic vulvar breast gland: a common neoplasm in an uncommon site. Pathologica. 2014;106(1):32-4.

6. Singh S, Mishra AK, Tewari S, Kumar S. Bilateral axillary masses mimicking as accessory breast with multiple fibroadenoma and bilateral gigantomastia in HIV-positive patient. BMJ Case Rep. 2013;2013. doi:10.1136/bcr-2013008563 